\title{
Simulação de textura de lesão cariosa para treinamento pré-clínico
}

\author{
Antônio Fernando Monnerat*; Carolina Cadette**; Aline Borges Luiz Monnerat***; Carolina Guedes
}

Barquete ${ }^{* * * * *}$

* Professor Associado do Departamento de Dentística, FO-UERJ

** Especialista em Odontopediatria, FO-UERJ

*** Doutora em Periodontia, FO-UERJ

**** Especialista em Dentística, FO-UERJ

Recebido em 21/11/2016. Aprovado em 08/02/2017.

\begin{abstract}
RESUMO
O objetivo deste estudo foi apresentar uma metodologia simples para a simulação de duas texturas de cárie em dentina em dentes de acrílico no treinamento pré-clínico. A proposta de desenvolvimento desta metodologia surgiu da necessidade de, durante o treinamento de remoção de cárie seletiva (parcial) por profissionais que atuavam em saúde pública, melhorar a compreensão em termos teóricos e práticos do que seriam as camadas da lesão cariosa em dentina: amolecida, em lascas, firme e endurecida. A metodologia permite identificar qual camada deve ser removida completamente, qual deve ser removida parcialmente e qual deve ser mantida intacta. $\mathrm{O}$ sucesso obtido no treinamento de mais de 1000 profissionais capacitados em diferentes prefeituras brasileiras foi o incentivo para a redação deste artigo, descrevendo detalhadamente a metodologia.

Descritores: Educação Odontológica. Materiais de Ensino. Odontologia Restauradora. Cariologia.
\end{abstract}

\section{INTRODUÇÃO}

Durante os anos 1990, a Odontologia sofreu forte impacto com as mudanças na forma de remover a lesão cariosa. O conceito de cárie deixou de ser descrito como lesão e passou a ser definido como uma doença multifatorial ${ }^{1}$.
Descobertas científicas de como as bactérias causadoras da doença promovem a desmineralização da estrutura dental ${ }^{2}$ e o desenvolvimento de novos sistemas adesivos e materiais restauradores capazes de selar a restauração ${ }^{3,4,5}$ também contribuíram para essa mudança de 
paradigma.

A odontologia minimamente invasiva $^{6}$ surgiu justamente da união da Cariologia com os materiais odontológicos adesivos modernos, como o cimento de ionômero de vidro e os sistemas adesivos resinosos. Além disso, alguns índices odontológicos também foram desenvolvidos, classificando, de forma diferente aos tradicionais CPOD e CPOS, as superfícies dentárias quanto à atividade ou não de doença cárie. Um destes índices, o critério $\mathrm{Nyvad}^{7} \mathrm{faz}$ menção a lesões ativas e inativas, além de considerar também lesões cavitadas e não cavitadas.

Os estudantes de Odontologia, durante a fase pré-clínica do curso, se deparam com treinamentos de preparos muitas vezes complexos, como preparos para restaurações metálicas, restaurações em cerâmica pura e metalocerâmica. Contudo, as restaurações diretas, principalmente as confeccionadas com resina e com cimento de ionômero de vidro, não têm uma forma de preparo estabelecida, sendo seu tamanho, profundidade e envolvimento de faces dentárias decorrente da extensão da lesão cariosa. As cavidades desenvolvidas pelos fabricantes de dentes de acrílico utilizados em laboratório são muito pouco condizentes com as lesões encontradas em pacientes, principalmente nos casos de lesões incipientes e lesões ocultas. Além disso, estas cavidades não proporcionam a noção de como o tecido cariado é encontrado, sua textura e dureza nas diferentes profundidades, dificultando o treinamento de remoção de cárie, técnica aprendida nas aulas teóricas de Cariologia e Dentística.

Alguns produtos, como o Lear-a-Prep II $^{8}$ (Whip Mix Corporation, Louisville, USA) e o Cavidrill $^{9}$ (Vivadent, Schaan, Liechtenstein) tentam dar esta noção ao aluno, contudo a simulação oferecida não diferencia as diferentes texturas de dentina presentes na lesão cariosa.
Estes dispositivos são superfícies planas que em nada se assemelham às cavidades cariosas de dentes humanos.

Outro aspecto importante a ser destacado é que a legislação brasileira criou leis específicas que dificultam a utilização de dentes humanos extraídos, os quais, se mantidos em meio úmido, poderiam simular as referidas condições. Mesmo assim, a utilização de dentes humanos sempre foi difícil, visto que os professores não tinham à sua disposição um padrão constante de lesão cariosa em todos os dentes.

Diante desse quadro, fica evidente a necessidade de se pensar em novos métodos e materiais para treinamento quanto à remoção de dentina cariada, permitindo simular diferentes texturas encontradas nas lesões de cárie, proporcionando treinamento pré-clínico padronizado, mais próximo da realidade clínica que o aluno encontrará em pouco tempo.

Este artigo relata a experiência dos autores com uma metodologia simples para simulação de duas texturas de cárie em dentina em dentes de acrílico.

\section{RELATO DE EXPERIÊNCIA}

A nomenclatura e as consistências aqui apresentadas são baseadas na terminologia de lesões de cárie proposta por Innes et al. (2016) ${ }^{9}$ : dentina amolecida, dentina em lascas, dentina firme e dentina endurecida.

Três dentes de acrílico da arcada superior de um manequim foram utilizados para demonstração, sendo um anterior (21) e dois posteriores (16 e 26). Para o desenvolvimento das camadas da lesão artificial de cárie foram utilizados os materiais que constam do quadro 1 .

Os três dentes foram preparados com broca esférica diamantada 1014 para que apresentassem lesão cariosa oculta ou incipiente em classe I, II e III, respectivamente. Um acesso proximal foi realizado de forma a preparar uma cavidade, 
inicialmente sem acesso oclusal. Nos casos de oclusais e dos sulcos principais e secundários, cavidades classes I e II, a broca removeu o sem, contudo, ampliar o acesso proximal (figura acrílico imediatamente abaixo das vertentes 1).

Quadro 1. Materiais utilizados para a simulação de textura das camadas de dentina cariada.

\begin{tabular}{|c|c|}
\hline Dentina cariada amolecida & Dentina cariada em lascas \\
\hline $\begin{array}{c}\text { Serragem (pó de madeira) fina } \\
\text { Vaselina sólida }\end{array}$ & $\begin{array}{c}\text { Cera para escultura macro marfim } \\
\text { Gesso tipo IV }\end{array}$ \\
\hline $\begin{array}{l}\text { Para cada porção de serragem deve-se utilizar } \\
\text { aproximadamente } 1 / 3 \text { da quantidade de vaselina, de forma } \\
\text { que essa seja a liga para que a serragem adquira uma } \\
\text { consistência de pasta homogênea, com textura pastosa o } \\
\text { suficiente para ser acomodada sobre o dente. }\end{array}$ & $\begin{array}{l}\text { Uma fina camada de gesso deve recobrir um cubo de cera } \\
\text { marfim, em quantidade suficiente para mudar sua coloração. } \\
\text { Um esculpidor do tipo gotejador aquecido mistura os } \\
\text { materiais para ser aplicado sobre o dente. Após o } \\
\text { resfriamento da cera, essa terá coloração amarelo- } \\
\text { amarronzada mantendo sua consistência inicial. }\end{array}$ \\
\hline
\end{tabular}
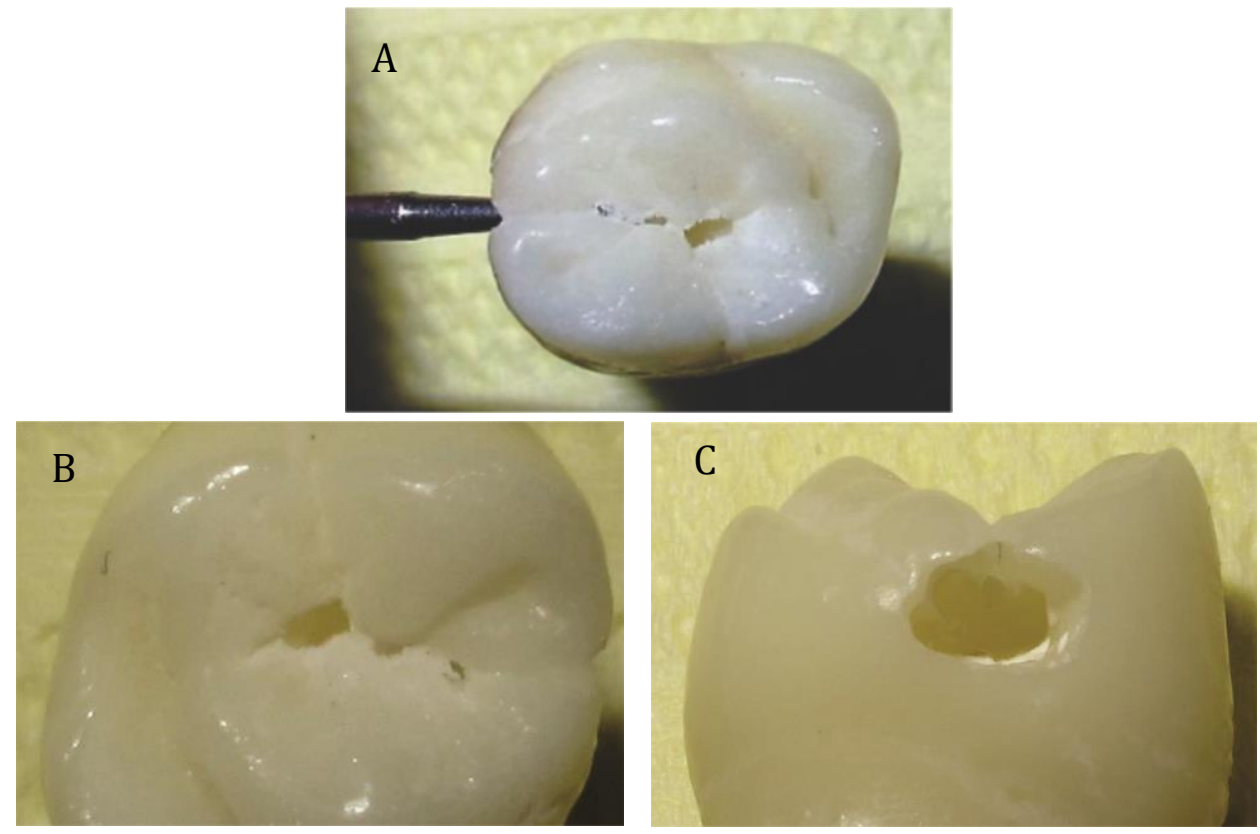

Figura 1 - Desgaste do dente realizado com broca 1014 para cavidades do tipo classe I (A, B) e classe II (C).

$\mathrm{Na}$ cavidade classe II, a proximal foi que a crista marginal do dente seja bem desgasdesgastada, estendendo o preparo para vestibular, tada, ficando com aspecto de "casca de ovo", para lingual e cervical. Nesse processo, é importante que o processo de fratura da parede proximal 
fique mais fácil durante o treinamento laboratorial. A extensão deste preparo pode variar de acordo com o tamanho da cavidade pretendida (figura 2). Tanto para a cavidade classe I e classe
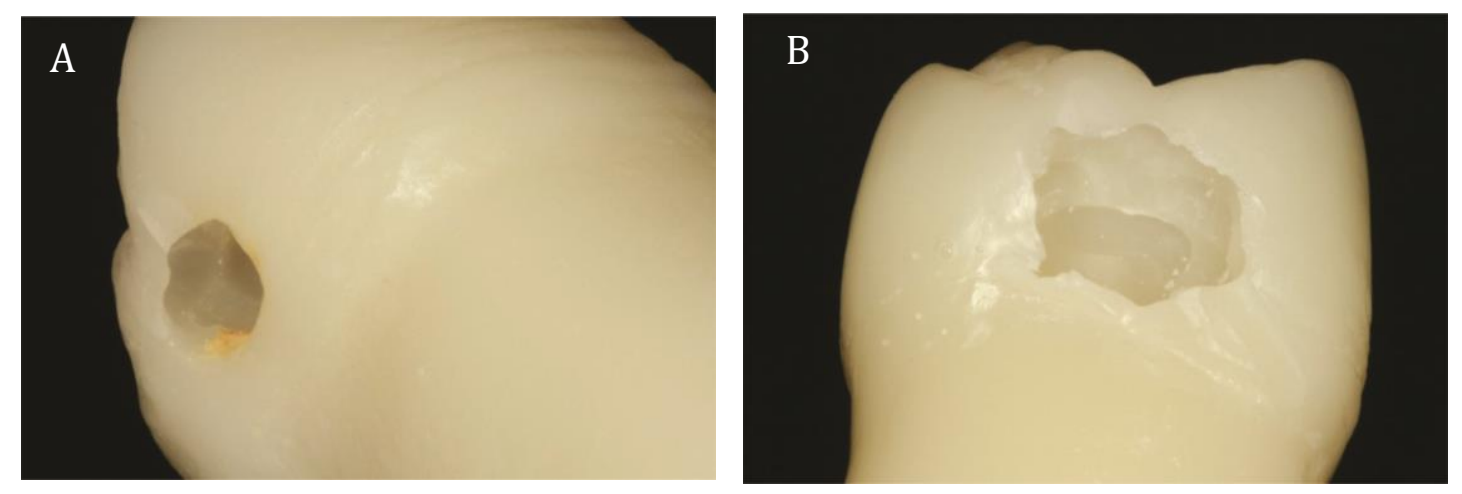

Figura 2 - Aspecto das paredes da cavidade em "casca de ovo" para confecção de cavidade do tipo classe II (A e B).
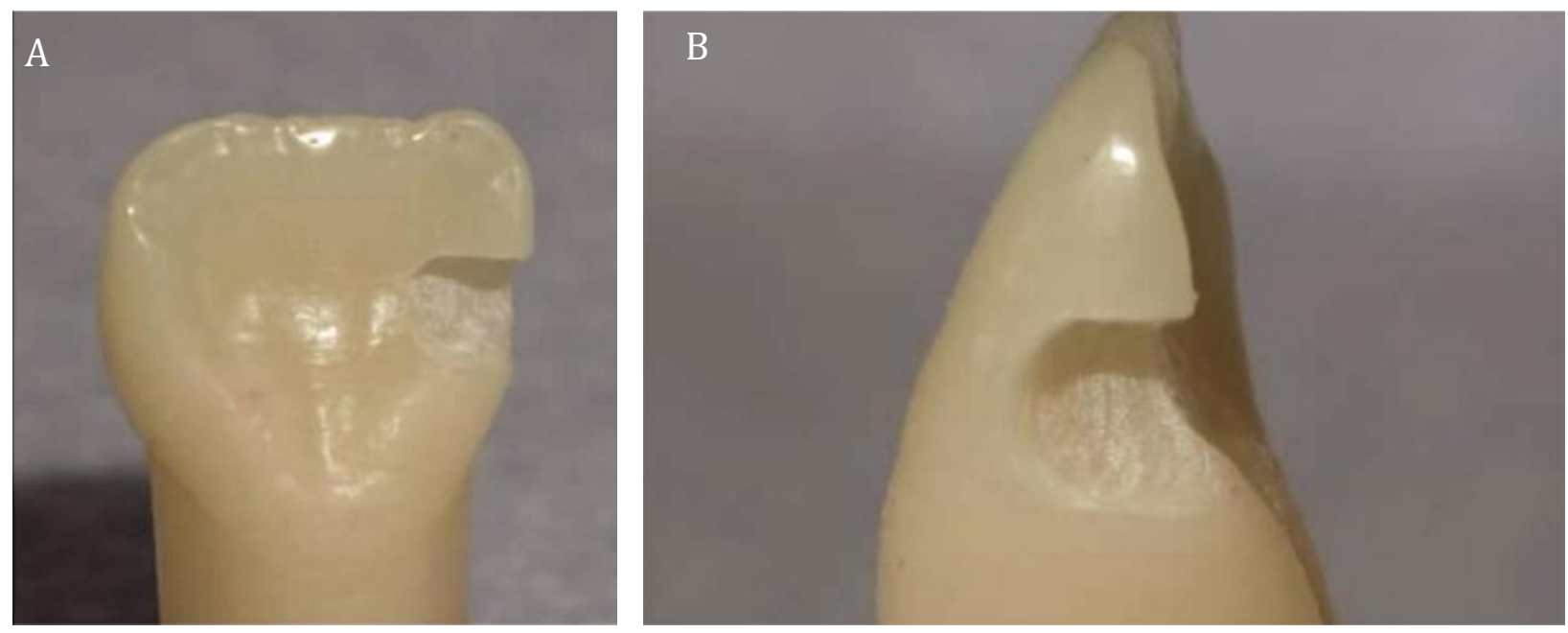

Figura 3 - Aparência das cavidades classe III (A e B).

Após a confecção da cavidade, as camadas de dentina cariada foram aplicadas cuidadosamente. A textura de dentina cariada em lascas foi aplicada com um gotejador aquecido nas paredes pulpar e axial da cavidade pelo acesso proximal em uma espessura correspondente a $1 / 3$
II, um pequeno acesso também foi executado no sulco principal. Na cavidade classe III, o mesmo princípio foi aplicado, ampliando-se ao máximo a cavidade sem ampliar o acesso proximal (figura 3). 
porção a ser removida (figura 5).

Posteriormente, um pouco de adesivo foi inserido cuidadosamente no limite da cavidade, fechar o acesso proximal (figura 6).
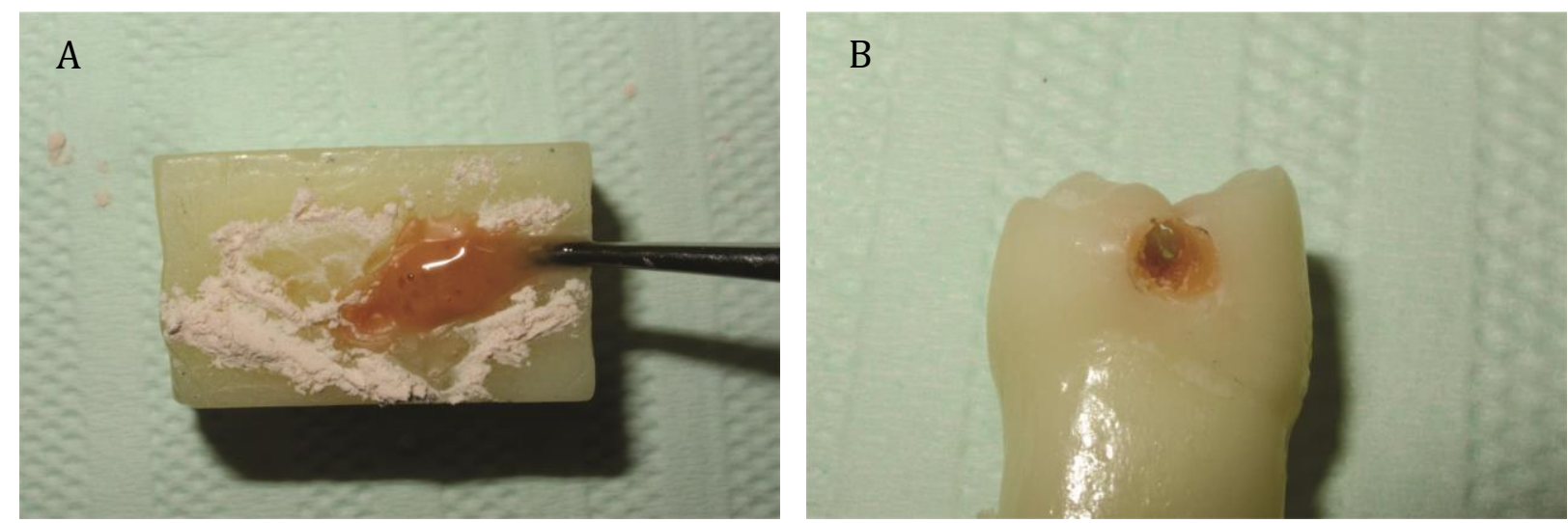

Figura 4 - Textura de dentina em lascas sendo preparada (A) e aplicada em 1/3 da cavidade com um gotejador (B).
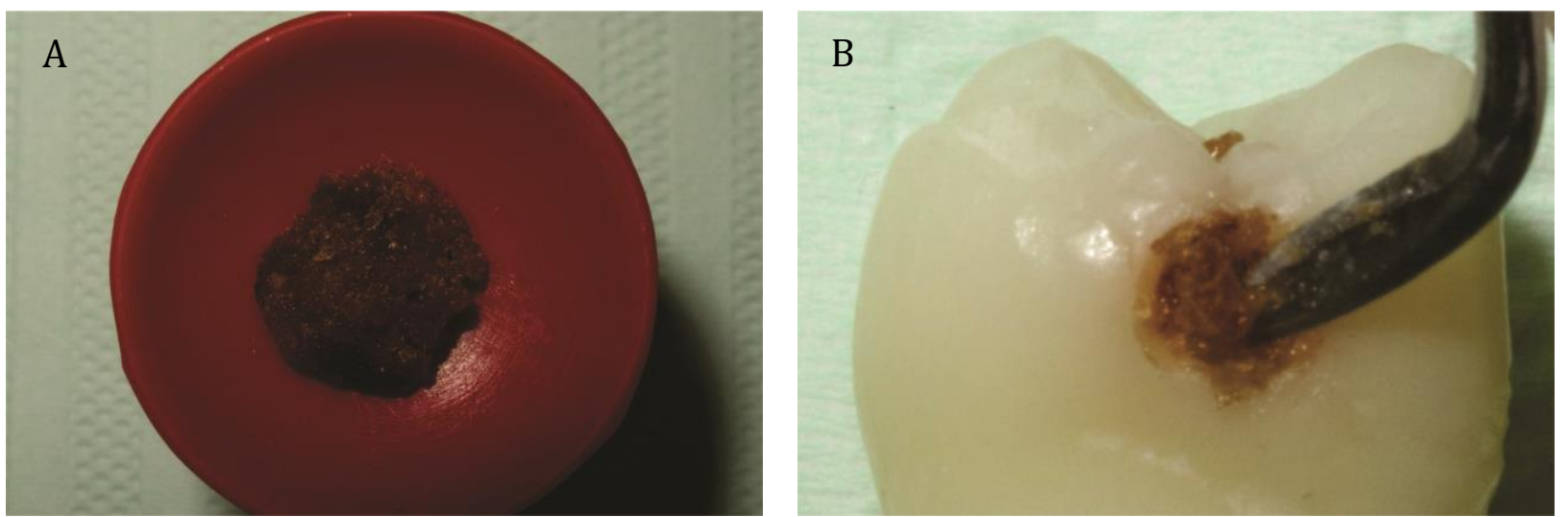

Figura 5 - Textura de dentina amolecida sendo preparada (A) e preenchimento do restante da cavidade (B).
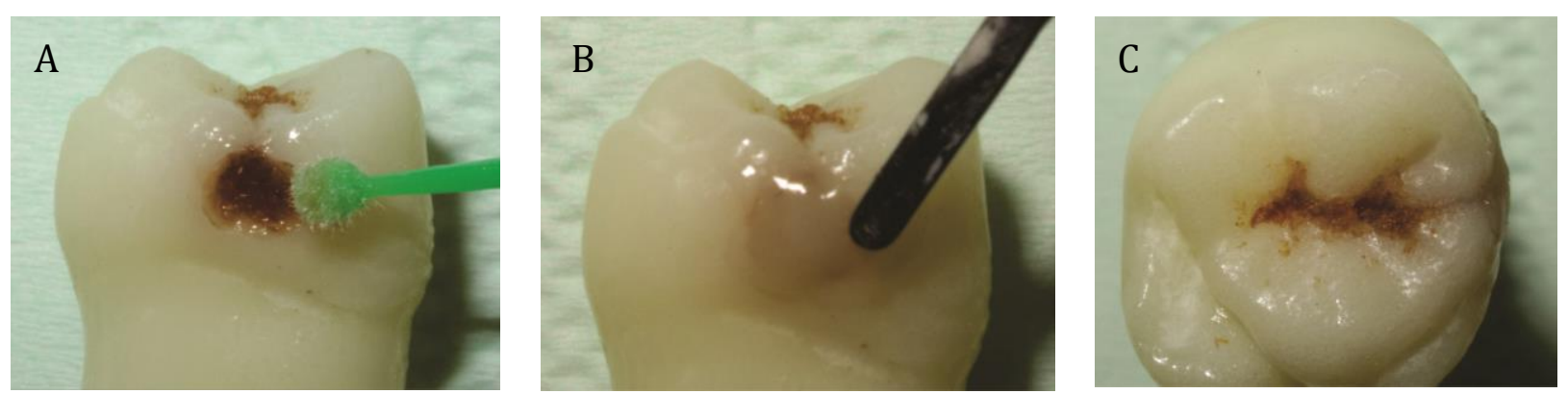

Figura 6 - Aplicação de adesivo com um microbrush (A), fechamento com resina fotopolimerizável da abertura proximal (B) e aspecto final (C). 
Os dentes foram mantidos em temperatura ambiente (entre $20^{\circ} \mathrm{C}$ e $24^{\circ} \mathrm{C}$ ), evitando calor excessivo ou frio extremo. A figura 7 mostra um dos dentes posicionado em um manequim para treinamento laboratorial, com o uso de um instrumento denominado "opener" ou "abridor", que atuará como uma broca, promovendo o

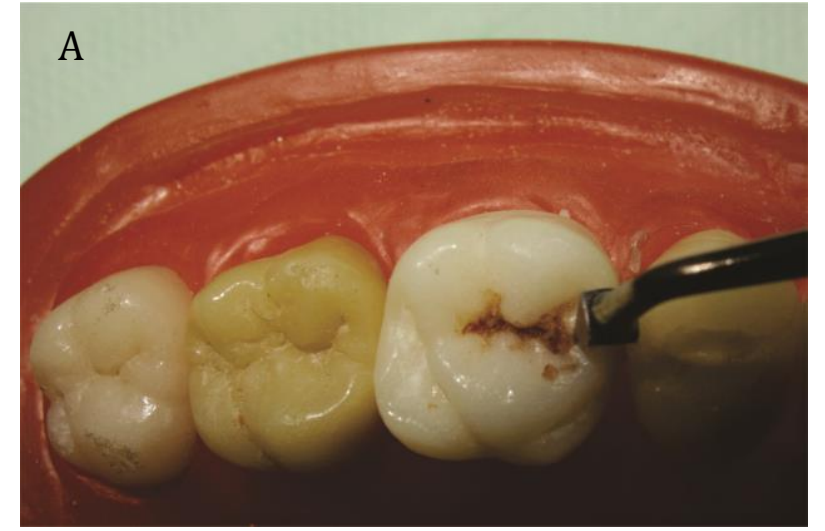

rompimento do esmalte e permitindo o acesso oclusal.

O uso de colheres de dentina de tamanhos variados também foi fundamental para ter acesso ao tecido cariado na junção amelodentinário e abaixo das cúspides e observar cuidadosamente a consistência desses tecidos.

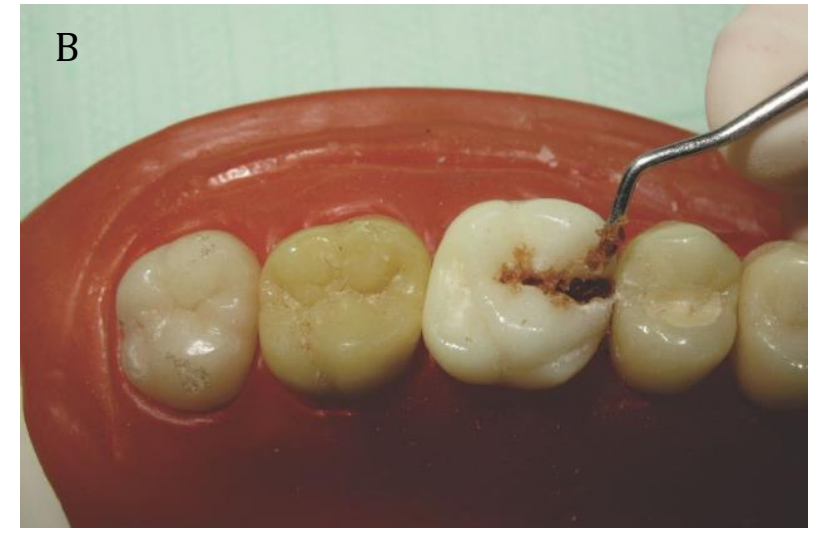

Figura 7 - Instrumento "opener" sendo apoiado sobre o esmalte para promover acesso à lesão (A) e remoção do tecido cariado amolecido com colher de dentina (B).

Esta metodologia foi aplicada no treinamento de mais de 1000 dentistas com experiência clínica variada (3 a 30 anos de formado) em tratamento restaurador atraumático (TRA). Informalmente, sem instrumentos de avaliação, foi possível constatar que os materiais apresentados se aproximam muito do que se encontra em procedimentos executados em clínica, podendo ser utilizado para diversos fins, como treinamento pré-clínico, cursos de restaurações diretas e atualização de profissionais que ainda utilizam a técnica de remoção total de tecido cariado.

\section{CONSIDERAÇÕES FINAIS}

A filosofia do TRA propõe tratamento sem a necessidade de instrumentos rotatórios. Durante o treinamento é possível remover todo o material que simula a dentina amolecida por meio de uma colher de dentina. Essa dentina é necrosada, decomposta e desorganizada, sendo facilmente removida, sem a necessidade de força adicional. A serragem, quando misturada com vaselina, adquiriu consistência de pasta similar a essa camada externa da lesão.

Já a dentina em lascas apresenta pequeno porcentual de bactérias e toxinas, é mais resistente à remoção com colheres de dentina e tem o potencial de remineralização, reorganização e reparação, por ainda possuir suas fibras colágenas. Portanto, não deve ser totalmente removida da cavidade, sendo sua consistência em "lascas" um indicativo de interrupção da remoção de tecido. A cera marfim, que foi utilizada como simulador dessa camada, apresentou grande semelhança de textura quando removida com colher de dentina.

A dentina em lascas é a camada de transição entre a dentina amolecida e a dentina firme. Esta e a camada subsequente, a dentina endurecida, 
são fisicamente resistentes à colher de dentina. Elas contêm tecido sadio, que deve ser preservado. Essas duas camadas, porém, não foram objeto de análise desse estudo e portando não foram simuladas. Isso se justifica pelo fato da maior dificuldade de reprodução dessa camada e a necessidade de distinguir as duas primeiras durante o treinamento de TRA. A própria consistência endurecida do dente de acrílico pode ser uma referência para o tecido hígido.

Durante treinamentos práticos de TRA observou-se que correta a remoção do tecido cariado foi um fator de dúvida por parte de estudantes e profissionais. Por isso, antes de iniciar os trabalhos clínicos, o treinamento laboratorial se faz necessário para esclarecer principalmente qual tipo de dentina deve ser removida e qual deve ser mantida, procurando ao máximo se assemelhar, também em consistência, à realidade.

\section{ABSTRACT \\ Carious lesion's texture simulation for pre- clinical training \\ The aim of this study was to present a simple method to simulate two dentin carious textures in acrylic teeth for preclinical training. The proposed development of this methodology arose from the need for the training of selective (partial) caries removal by professionals working in public health, improving the theoretical and practical understanding regarding the lesions' layers: soft, leathery, firm, and hard dentine. This method allows to identify which layer should be removed completely, which should be partially removed and what should be left intact. The success in training over 1000 professionals working at Brazilian Dental Public Health System was the incentive for writing this article, detailing the methodology.}

Descriptors: Dental Education. Teaching Materials. Restorative Dentistry. Cariology.

\section{REFERÊNCIAS}

1. Kidd E. The implications of the new paradigm of dental caries. J Dent. 2011 Dec;39(Suppl. 2):S3-8.

2. Ogawa K, Yamashita Y, Ichijo T, Fusayama T. The ultrastructure and hardness of the transparent layer of human carious dentin. J Dent Res. 1983 Jan;62(1):7-10.

3. Bjørndal L, Larsen T, Thylstrup A. A clinical and microbiological study of deep carious lesions during stepwise excavation using long treatment intervals. Caries Res. 1997; 31(6):411-7.

4. Maltz M, Oliveira EF, Fontanella V, Bianchi R. A clinical, microbiologic, and radiographic study of deep caries lesions after incomplete caries removal. Quintessence Int. 2002;33(2):151-9.

5. Handelman SL, Leverett DH, Solomon ES, Brenner CM. Use of adhesive sealants over occlusal carious lesions: radiographic evaluation. Community Dent Oral Epidemiol. 1981 Dec;9(6):256-59.

6. Elderton RJ \& Mjör IA. Changing scene in cariology and operative dentistry. Int Dent J. 1992 Jun;42(3):165-9.

7. Nyvad B, Machiulskiene V, Baelum V. Reliability of a new caries diagnostic system differentiating between active and inactive caries lesions. Caries Res. 1999 JulAug;33(4):252-260.

8. Boushell LW, Walter R,Phillips C. Learn-APrep II as a Predictor of Psychomotor Performance in a Restorative Dentistry Course. J Dent Educ. 2011 Oct;75(10): 1362-9.

9. Innes NP, Frencken JE, Bjørndal L, Maltz M, Manton DJ, Ricketts D, Van LanduytK, Banerjee A, Campus G, Doméjean S, Fontana M, Leal S, Lo E, Machiulskiene V,Schulte A, Splieth C, Zandona A, Schwendicke F. Managing carious lesions: consensus recommendations on terminology. Adv Dent Res. 2016 May;28(2):49-57.

Correspondência para:

Prof. Dr. Antônio Fernando Monnerat e-mail: antoniofmonnerat@gmail.com Boulevard 28 de setembro, 157 20551-030 Vila Isabel - Rio de Janeiro/RJ 